\title{
L'impact des styles d'apprentissage sur la performance des futurs enseignants du FLE en compréhension de l'oral
}

\section{* Dr. Chaïmaa Mohamed Tawfik Mohamed}

\section{Résumé}

Le but ultime de la présente étude était d'examiner l'impact qu'exercent les styles d'apprentissage des futurs enseignants du FLE sur leurs performances en compréhension de l'oral. Durant l'année académique 2014/ 2015, trente futurs enseignants du FLE à l'université d'Alexandrie ont répondu à un test d'écoute destiné à préparer les individus aux tests de performance (comme le DALF) et ont rempli un questionnaire fondé sur le modèle VAK de Fleming. Les résultats de l'analyse de variance à un facteur (ANOVA) met en évidence qu'il s'agit des différences statistiques significatives entre la performance en compréhension de l'oral des futurs enseignants du FLE ayant un style d'apprentissage auditif et la performance de ceux ayant un style visuel, en faveur des premiers. Les styles d'apprentissage s'avèrent donc un moyen efficace pour mieux prédire la performance en compréhension de l'oral chez les futurs enseignants.

Mots clés: Performance en compréhension de l'oral, styles d'apprentissage, modèle VAK de Fleming, style auditif, futurs enseignants du FLE.

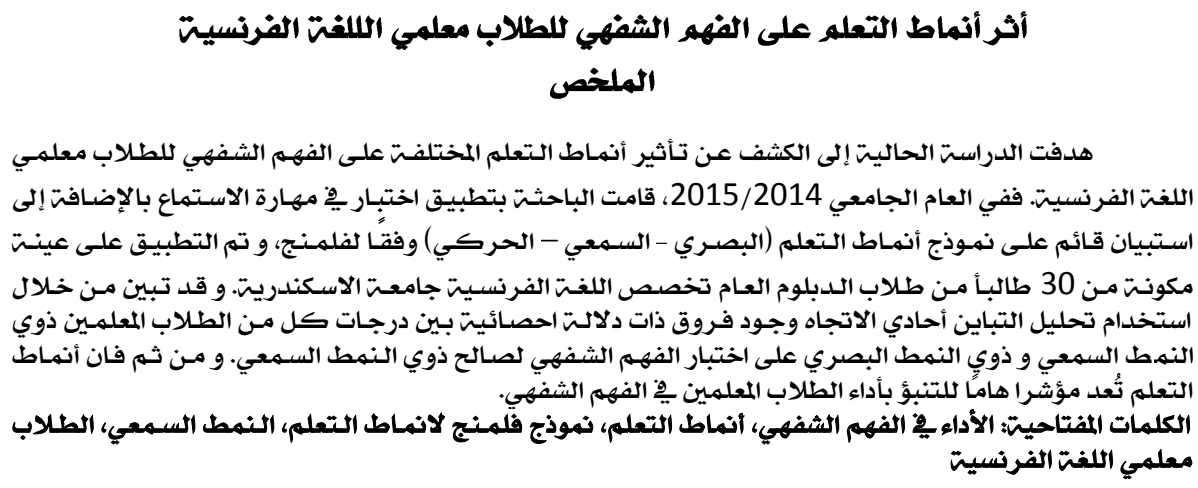

\section{Introduction}

Depuis de longues années, un intérêt croissant est attribué à l'apprentissage des langues étrangères. Celles-ci constituent un moyen indispensable pour assurer la communication entre les gens de par le monde, tout en leur garantissant une ouverture sur les

Maître de conférences - Département de curricula \& méthodologie- Faculté de Pédagogie - Université d'Alexandrie 
différents domaines de la vie. Il est incontestable que l'apprentissage de toute langue dépend essentiellement de la maîtrise de ses quatre habiletés langagières intrinsèquement liées, à savoir l'écoute, la parole, la lecture et l'écriture. C'est pourquoi l'admission des étudiants dans certains types de formations exige leur passation par un test standardisé (tels que le DELF, le DALF, le TOEFL, le IELTS, etc.) visant à mesurer leur niveau de performance au niveau des quatre habiletés langagières. A titre d'exemple, au cours de ces tests, le candidat doit manifester ses aptitudes à comprendre un texte écrit, comprendre des phrases et de courtes conversations et s'exprimer oralement et par écrit.

Il faut bien souligner que de nombreux étudiants éprouvent de graves difficultés à répondre à certaines questions de ces tests, ce qui aura, certes, un impact négatif sur leurs notes. A titre d'illustration, quelques étudiants peuvent répondre aisément aux questions de compréhension écrite quoiqu'ils aient du mal à répondre à celles de compréhension orale, d'autres n'éprouvent aucune difficulté à répondre aux questions de production écrite, ce qui n'est pas le cas pour la production orale ou vice versa. Cet exemple met l'accent sur l'existence des différences individuelles au niveau de la performance des étudiants. En effet, cette performance, qui se manifeste indubitablement via les notes des apprenants, est le fruit de divers facteurs dont le plus important est, selon Chartier (2003: 9), les styles d'apprentissage que préfèrent les individus. La notion de "styles d'apprentissage" suggère que chaque individu a sa propre façon de percevoir, stocker, traiter et restituer l'information en fonction de son style préférentiel, ce qui le distingue d'autrui (Raven et al., 1993: 40; Chartier, 2003: 9).

Dans la présente étude, la chercheuse tient à examiner les styles d'apprentissage des futurs enseignants du FLE tout en découvrant leur impact sur le niveau de performance de ceux-ci en ce qui concerne la compréhension de l'oral. II est vraisemblable que la compréhension de l'oral (l'écoute) est l'une des habiletés langagières indispensables à l'apprentissage des langues en général et celui des langues étrangères en particulier. Néanmoins, cette habileté est tant 
négligée dans le processus de l'enseignement des langues comme l'affirment Gagnon et al. (2017). De plus, un bon nombre d'étudiants éprouvent des difficultés à répondre aux questions portant sur la compréhension orale.

\section{Problématique de l'étude}

La problématique de l'étude actuelle réside en la faible performance des futurs enseignants de FLE à l'université d'Alexandrie en répondant à des questions de compréhension orale. C'est pourquoi il s'avère nécessaire de mettre l'accent sur les styles d'apprentissage que préfèrent ces futurs enseignants et qui peuvent sous-tendre leurs performances en compréhension orale.

On peut formuler cette problématique à partir des trois questions suivantes:

1) Quels sont les styles d'apprentissage des futurs enseignants du FLE inscrits au diplôme général?

2) Quelle est la performance des futurs enseignants du FLE inscrits au diplôme général en compréhension de l'oral?

3) Quel impact exercent les différents styles d'apprentissage des futurs enseignants du FLE inscrits au diplôme général sur leurs performances en compréhension de l'oral?

\section{Objectifs de l'étude}

La présente étude vise à:

1. examiner l'effet des styles d'apprentissage des futurs enseignants du FLE sur leurs performances en compréhension de l'oral.

2. éveiller la conscience des futurs enseignants du FLE autour de leurs propres styles d'apprentissage pour qu'ils l'éveillent ultérieurement chez leurs apprenants.

3. sensibiliser les futurs enseignants $d u$ FLE à travailler davantage leurs styles d'apprentissage moins dominants afin de d'en profiter dans les différentes situations d'apprentissage. 


\section{Importance de l'étude:}

L'étude actuelle acquiert son importance du fait qu'elle:

1. attire l'attention des responsables pour prendre en considération l'impact qu'exercent les différents styles d'apprentissage sur le niveau de performance des individus dans les tests standardisés.

2. conscientise les futurs enseignants du FLE sur la nécessité de varier leurs méthodes d'enseignement pour convenir à la diversité de styles d'apprentissage dont jouissent les apprenants.

\section{Limites de l'étude}

Il s'agit de/d':

1. trente étudiants inscrits au diplôme général, section française, à la faculté de Pédagogie d'Alexandrie en 2014/ 2015.

2. un questionnaire portant sur le modèle VAK (visuel - auditif et kinesthésique) des styles d'apprentissage de Fleming.

3. la performance des futurs enseignants du FLE au niveau de la compréhension de l'oral.

4. un test de compréhension orale.

5. l'analyse de variance à un facteur (ANOVA) comme technique statistique visant à déterminer si la performance des futurs enseignants du FLE à un test de compréhension de l'oral diffère selon leurs styles d'apprentissage.

\section{Terminologie de l'étude}

- Les styles d'apprentissage constituent le mode de fonctionnement préféré par l'apprenant au cours d'un contexte d'apprentissage. Ce mode, qui émerge des représentations que l'apprenant a de lui-même et de la tâche à accomplir, semble évident par "l'expression de préférences pour certaines manières de faire et par le choix de conduites et de stratégies d'apprentissage particulières auxquelles l'apprenant s'identifie" (Chevrier et al. 2000b).

- La compréhension de l'oral: C'est un processus mental complexe qui consiste à recevoir des sons (des entrées) et à les diviser en unités linguistiques (morphèmes et mots). La 
récupération du sens de ces unités linguistiques se fait à partir de la mémoire à long terme aussi bien que les connaissances préalables que possède l'auditeur (Buck, 2001; Rost, 2011).

\section{Cadre théorique}

Depuis de longues années, dû à l'engouement tant pour la psychologie cognitive que pour les neurosciences, des chaires et des sociétés savantes ont été créées et des colloques se tiennent afin de mettre en exergue le concept des styles d'apprentissage (Gaussel, 2013). Un survol des écrits portant sur cette notion met en relief tant la pluralité que la diversité de ses définitions. Or, dans cette étude, il ne s'agit point de présenter de façon exhaustive toutes les définitions concernant cette notion. C'est pourquoi la chercheuse tentera de donner un bilan ou une esquisse de quelques-unes de ces définitions.

- Ahmad (2011) et Barmeyer (2007): les styles d'apprentissage renvoient aux manières naturelles, habituées et privilégiées dont un individu se sert en vue d'acquérir un savoir.

- Brown (2000): ils se réfèrent à la manière individuelle à percevoir et à traiter les informations dans des situations d'apprentissage.

- Rieben (2000): ils désignent les manières avec lesquelles les personnes réfléchissent, apprennent et accomplissent différemment des tâches déterminées.

- Cornett (1983): la notion en question représente un ensemble cohérent de comportements pour agir et se comporter dans un contexte d'apprentissage. Ces comportements varient d'une personne à l'autre.

A partir des définitions mentionnées ci-dessus, maintes conclusions sont à tirer:

Premièrement, bien que le concept de styles d'apprentissage ait été sujet d'une multitude de recherches au cours des dernières décennies, il n'a pas été nettement défini (Chevrier et al., 2000a:510). A cet égard, Gilakjani (2012: 105) affirme que les définitions diverses reflètent par excellence la diversité des points de vue des auteurs. Deuxièmement, le concept de styles d'apprentissage est 
considéré soit comme un processus de traiter les informations et d'apprendre, soit comme une manière distincte et plus ou moins constante d'agir dans un contexte d'apprentissage (Chevrier et al., 2000a: 6; Frayssinhes, 2011: 81). En d'autres mots, les différentes définitions mettent l'accent sur le concept de style d'apprentissage tantôt comme un processus tantôt comme un produit.

Or, en dépit de ces définitions diverses, elles jouissent, toutes, d'un dénominateur commun, à savoir que les styles d'apprentissage diffèrent d'une personne à l'autre. Ils contribuent donc à caractériser l'individu.

Dans cette étude, la chercheuse adopte la définition susmentionnée de Chevrier et al. (2000b: 62) qui semble, à notre avis, la plus adéquate pour deux raisons: d'une part, elle a mis en relief le style d'apprentissage en tant que processus interne qui se manifeste sous formes de comportements et de conduites préférés par l'individu (un produit). D'autre part, cette définition a tenu compte que le style d'apprentissage varie non seulement d'une personne à l'autre, mais aussi chez une même personne en fonction de la tâche à achever. Autrement dit, les styles d'apprentissage ne sont pas fixes (ce point sera traité plus tard) dès la naissance, par contre ils peuvent être modifiés à la lueur du contexte auquel la personne est soumise.

D'ailleurs, afin de mieux cerner la notion de styles d'apprentissage, il s'avère utile de traiter brièvement des notions très proches telles que les stratégies d'apprentissage, le style cognitif et les intelligences multiples, tout en mettant l'accent sur le rapport qu'elles entretiennent avec les styles d'apprentissage, notion primordiale dans cette étude.

En effet, les styles et les stratégies d'apprentissage sont deux concepts qui occupent une place culminante dans le domaine de la linguistique appliquée. Ils fournissent des facteurs fondamentaux au processus et au résultat de l'apprentissage. Selon Oxford (1990), une stratégie d'apprentissage désigne des actions spécifiques, des comportements, des étapes ou des techniques que l'apprenant utilise, dans des situations d'apprentissage en vue d'accomplir une 
tâche, de résoudre un problème, etc. A cet égard, Hanachi- Ferhoune (2007: 15), Atlan (2000: 112-113) et Therer (1998: 10) insistent que ces actions sont délibérément choisies par l'apprenant. Ce qui indique que les stratégies d'apprentissage renvoient à des comportements conscients. Cependant, O'Malley et Chamot (1995: 2) dénotent qu'à force d'utiliser ces stratégies, elles peuvent devenir inconscientes donc automatiques.

Quant aux styles d'apprentissage, Cornett (1983: 9) les considèrent comme des approches générales (à titre d'exemple des manières globales/ analytiques ou auditives/visuelles) auxquelles les apprenants ont recours pour apprendre. De surcroît, d'aucuns affirment que les styles d'apprentissage font partie de la personnalité de l'individu (Wang, 2015). Ainsi, on peut constater que les styles d'apprentissage est un concept très large alors que celui de stratégies d'apprentissage est plus restreint puisqu'il se réfère à des opérations plus détaillées.

En outre, selon Flessas (1997), le style cognitif fournit "la façon propre à chacun de percevoir, d'évoquer, de mémoriser et donc de comprendre l'information perçue à travers les différentes modalités sensorielles qui sont à sa disposition face à une connaissance nouvelle". Shi (2011: 20) ajoute que cette notion est classée sous forme de deux extrémités: dépendant/indépendant, analytique/ global, réflectif/ impulsif, etc. D'après Dörnyei et Skehan (2003: 602), ce qui distingue le style cognitif du style d'apprentissage est que le premier est centré uniquement sur la manière de traiter l'information, alors que le second ne s'arrête pas à ce stade; il le dépasse pour se rapporter à tous les aspects de l'apprentissage. A cet égard, Bouchafa (2009) affirme que le style cognitif se limite aux facteurs cognitifs, tandis que le style d'apprentissage s'étend à d'autres éléments: affectifs, psychologiques et sociologiques. On peut donc constater que le style cognitif est une partie intégrante du style d'apprentissage.

Par ailleurs, vers la fin du siècle précédent, le concept de style d'apprentissage a été mis en rapport avec un autre concept, à savoir I'intelligence. Selon Le Robert Micro Poche (1997: 688), I'intelligence 
constitue la faculté mentale non seulement de connaître et de comprendre les choses et les faits mais également de découvrir les liens entre eux. D'ailleurs, dans son ouvrage intitulé Frames of mind, Gardner estime qu'il ne s'agit point d'une seule intelligence mais de plusieurs, ce qui a donné naissance à sa fameuse théorie des intelligences multiples qui reflète par excellence les façons dont se sert l'esprit pour créer son rapport avec le monde, avec les autres et avec lui-même (Torresan, 2010: 13 ; Denig, 2004: 97-98). Selon cette théorie, considérée comme une importante contribution dans les sciences cognitives, il est question de neuf types d'intelligence liés à des zones différentes du cerveau. Ces intelligences sont: I'intelligence verbale/ linguistique, l'intelligence logico - mathématique, l'intelligence musicale/ rythmique, l'intelligence kinesthésique/ corporelle, l'intelligence visuelle/ spatiale, l'intelligence interpersonnelle, l'intelligence intrapersonnelle, l'intelligence naturaliste et l'intelligence existentielle. II est important de signaler que ces types d'intelligence se présentent comme un ensemble: "travaillant en parallèle, dont chacun contribue au succès de la tâche entreprise grâce à sa potentialité spécifique (Torresan, 2010: 13 - 27).

Gardner (1999) affirme que toute personne jouit de ces neuf types d'intelligences, cependant, il y en a un qui domine chez elle et c'est par celui-ci qu'elle apprend le mieux. Le tableau suivant met en relief en quoi les intelligences multiples se distinguent- elles des styles d'apprentissage (Özgen et al., 2011: 171; Prashnig, 2005: 8,9): 
Tableau 1 Une comparaison entre les intelligences multiples et les styles d'apprentissage

\begin{tabular}{|c|c|}
\hline Les intelligences multiples & Les styles d'apprentissage \\
\hline $\begin{array}{l}\text { C'est un cadre théorique pour } \\
\text { définir, comprendre, évaluer ou } \\
\text { développer les différents facteurs } \\
\text { contribuant à l'intelligence humaine. }\end{array}$ & $\begin{array}{l}\text { La manière que préfère l'individu } \\
\text { pour se concentrer, retenir ou se } \\
\text { mémoriser une information. }\end{array}$ \\
\hline $\begin{array}{l}\text { Elles sont centrées sur ce que } \\
\text { l'individu apprend (le produit). }\end{array}$ & $\begin{array}{l}\text { Ils sont centrés sur la manière } \\
\text { d'apprendre (le processus). }\end{array}$ \\
\hline $\begin{array}{l}\text { Elles supposent que les personnes } \\
\text { apprennent d'une manière intuitive. }\end{array}$ & $\begin{array}{l}\text { Ils estiment que certaines personnes } \\
\text { apprennent d'une manière intuitive, } \\
\text { tandis que d'autres ont besoin } \\
\text { d'être guidés. }\end{array}$ \\
\hline $\begin{array}{l}\text { Les adeptes de cette théorie } \\
\text { préconisent un changement dans la } \\
\text { méthodologie utilisée en classe. } \\
\text { Celle-ci doit mettre l'accent sur les } \\
\text { talents des apprenants de la même } \\
\text { manière et simultanément. }\end{array}$ & $\begin{array}{l}\text { Il est recommandé d'exploiter les } \\
\text { différentes ressources éducatives } \\
\text { tout en assurant leur harmonie avec } \\
\text { les diverses manières qui } \\
\text { permettent aux individus de mieux } \\
\text { apprendre. }\end{array}$ \\
\hline
\end{tabular}
intelligences et les styles d'apprentissage se distinguent-ils? L'exemple ci-dessous mettra en exergue la réponse à cette question. Supposons les deux situations suivantes: un enseignant se sert d'une musique de fond au cours d'une activité d'écriture; tandis qu'un autre invite ses apprenants à faire une composition écrite tout en s'inspirant d'un morceau de musique. Dans le premier cas, la musique de fond représente un stimulus périphérique qui sert uniquement à provoquer des associations personnelles. En effet, la musique qui accompagne un exercice de rédaction n'est pas une activité stimulant l'intelligence musicale, mais une activité appréciée par les apprenants dont le style d'apprentissage est musical. Par contre, dans le second cas, où la musique est un stimulus intermédiaire mis au premier plan, l'apprenant doit tisser, d'une manière raisonnée, le lien entre le code linguistique et le code musical (Torresan, 2010: 19). On constate donc que l'activité du premier cas renvoie au style d'apprentissage, tandis que la seconde 
concerne l'intelligence musicale, l'une des intelligences multiples. Ainsi, on peut conclure qu'il s'agit d'une ligne très fine entre les styles d'apprentissage et les intelligences multiples: cette ligne définit le stimulus sur la base du fait qu'il exerce ou non un effet cognitif. En d'autres termes, il existe un continuum entre les styles d'apprentissage et les intelligences multiples: le style d'apprentissage devient un type d'intelligence lorsque le stimulus exerce un effet cognitif (Torresan, 2010: 19-21).

D'ailleurs, il faut bien souligner que si les styles d'apprentissage et les intelligences multiples sont deux notions distinctes, cela n'empêche pas la présence de similitudes entre elles. Parmi ces similitudes citons: en premier lieu, les deux concepts en question ont pour point de mire l'apprenant et son apprentissage, ce qui symbolise un penchant complètement différent de l'enseignement traditionnel. L'apprenant n'est plus donc un récepteur passif, et l'enseignant n'est plus l'unique détenteur du savoir. Celui- ci devient plutôt un facilitateur, un guide et un conseiller. En deuxième lieu, les deux notions prennent en considération les différences individuelles. De même, on ne peut pas nier la ressemblance concernant les différents types de chaque concept; par exemple: intelligence kinesthésique/style kinesthésique, intelligence spatiale/style spatial, intelligence musicale/style musical, etc. (Dunn et al., 2001; Guild, 1997; Silver et al., 1997).

De ce qui précède, il est donc indéniable que le style d'apprentissage constitue une notion complexe et controversée de par sa parenté avec une multitude de notions très proches.

Par ailleurs, les pédagogues adoptent deux points de vue opposés concernant les caractéristiques des styles d'apprentissage. D'une part, les innéistes estiment que le style d'apprentissage constitue une caractéristique immuable et figée. Ce point de vue est préconisé au sein des typologies de styles d'apprentissage fondées sur les différences hémisphériques du cerveau comme nous allons l'aborder plus tard. Selon cette vision, l'enseignant n'a qu'à fournir, aux apprenants, divers contextes qui conviennent parfaitement à 
leurs différents styles d'apprentissage. Son rôle n'est pas donc de les initier à d'autres manières de fonctionner (Frayssinhes, 2011: 79).

D'autre part, d'aucuns conçoivent les styles d'apprentissage en tant qu'une caractéristique non figée et évolutive. Ils estiment que les styles d'apprentissage se modifient à la lueur de l'exercice réclamé, de la compétence de l'apprenant, voire de son humeur (Torresan, 2010: 11). Selon cette optique, l'apprenant est capable d'adopter plusieurs styles d'apprentissage en fonction des circonstances.

Face à cette dichotomie, certains auteurs suggèrent une vision intermédiaire. A titre d'illustration, Aliweh (2011: 82) et Chartier (2003: 20) déclarent que les styles d'apprentissage sont, en général, stables de sorte qu'on peut les tenir en compte au cours d'une situation donnée pour décrire les comportements d'apprentissage d'un individu. Néanmoins, au cas où la situation d'apprentissage est modifiée, de façon significative, un individu peut varier son style préférentiel d'apprentissage dans le but de s'accommoder à la situation. C'est pourquoi Smith et Dalton (2005: 5) affirment que toute personne peut avoir une multitude de styles d'apprentissage en fonction de la situation et de la tâche d'apprentissage qu'elle assume. De ce qui précède, nous pouvons donc constater que l'individu doit être souple et ouvert d'esprit afin de choisir le style d'apprentissage adéquat à la situation rencontrée.

Par ailleurs, les styles d'apprentissage sont-ils innés ou appris? Selon de nombreux pédagogues tels qu'Arthur (1993: 8), la majorité de nos styles d'apprentissage sont appris, au cours de notre enfance, à travers l'entourage avec qui nous agissons régulièrement. Cet entourage nous assure une connaissance non seulement autour du monde où nous vivons, mais aussi autour de la manière d'apprendre, à savoir le style d'apprentissage, ce qui aura un effet considérable sur le nôtre. Arthur (1993: 8,9) affirme, d'emblée, que de nouveaux styles d'apprentissage peuvent être appris surtout durant les premières années à l'école lorsque le style d'apprentissage encouragé par l'enseignant diffère de celui stimulé par les parents. 
En outre, il est important que l'apprenant aussi bien que l'enseignant soient conscients de leurs propres styles d'apprentissage. D'une part, lorsque l'apprenant est conscient de son propre style d'apprentissage, il sera apte de déterminer ses points forts et ses points faibles en vue de renforcer les premiers et de remédier aux seconds, ce qui augmente sa motivation pour apprendre et le rend capable de s'adapter aux différentes situations qu'il confronte. De surcroît, cette prise de conscience contribue à améliorer la qualité de l'apprentissage et à le mieux gérer. L'apprenant sera, en conséquence, responsable de son apprentissage et par la suite il lui attribuera un sens. On peut donc parler d'un apprentissage permanant (Karthigeyan \& Nirmala, 2013: 134; Gilakjani \& Ahmadi, 2011: 471; Gülbahar \& Alper, 2011: 270; Penger \& Tekavčič, 2009: 16; Cox, 2008: 1; Coffield, 2004; Tremblay \& Paradis, 2001: 3).

Or, il faut signaler que lorsque l'individu témoigne une conscience de son propre style d'apprentissage, cela ne veut point dire qu'il sera prisonnier d'une certaine étiquette. Cette prise de conscience lui offrira, par contre, l'opportunité de réfléchir sur sa propre manière de penser et de s'ouvrir sur de nouvelles façons d'apprendre pour avoir un apprentissage plus efficace. Cette réflexion métacognitive pourra, en conséquence, maximiser ses chances d'apprentissage (Fleming \& Baume, 2006: 7; Lavine \& Fiala, 2006: 10). C'est pourquoi de nombreuses études telles que l'étude de Zainol Abidin et al. (2011) affirment que la connaissance des individus de leurs styles d'apprentissage constitue l'un des facteurs jouant un rôle crucial dans leur réussite académique.

D'autre part, si l'enseignant est tout à fait conscient de son propre style d'apprentissage qui ne ressemble pas forcément aux styles d'apprentissage de ses apprenants, il sera donc en mesure d'individualiser son enseignement. En d'autres termes, le fait de connaître que les individus n'apprennent pas tous de la même manière éveille chez l'enseignant l'importance de diversifier ses méthodes d'enseignement, les activités et les types d'évaluation qu'il utilise en concordance avec les besoins des différents types d'élèves, 
ce qui contribuera à accroître l'engagement et la réussite de chacun d'eux. Par conséquent, il réussira à satisfaire à une gamme élargie de goûts et d'intérêts au sein de la classe (Torresan, 2010: 12; Franzoni \& Assar, 2009: 15; Dennis, 2002). A cet égard, Kassaia (2007: 54) et Oxford (2003: 2- 3) avouent qu'une harmonie entre les styles d'apprentissage et les méthodes d'enseignement joue un rôle primordial à améliorer la performance de l'apprenant tout en augmentant sa confiance en soi-même et en diminuant son anxiété au cours de l'apprentissage. De plus, de nombreuses études, à l'instar de celle de Matthews (1996) et celle d'Oxford \& Lavine (1992), ont assuré qu'un style d'enseignement, qui tient compte des divers styles d'apprentissage, garantit une progression au niveau académique des apprenants de tous les cycles. D'ailleurs, la prise de conscience que manifeste l'enseignant, face aux styles d'apprentissage de ses étudiants, permet également une interaction entre l'enseignant et les apprenants; celle-ci est basée essentiellement sur le respect des différences (Matthews, 1996). Il s'agit donc d'un cours dynamique où les apprenants sont engagés dans le processus de leur apprentissage, ce qui augmente, selon Lavine \& Fiala (2006: 11), le potentiel scolaire de chaque apprenant. Ainsi, on constate que, dans ce cadre, le rôle qu'assume l'enseignant va au delà de la simple transmission des connaissances aux apprenants. Celui-ci exerce de nouveaux rôles: il est un guide, un conseiller et un médiateur qui tient à assurer une concordance entre son style et sa méthode d'enseignement, d'un côté, et les différents styles de ses apprenants, d'un autre côté, afin d'assouvir leurs besoins.

Par ailleurs, en survolant les écrits portant sur les styles d'apprentissage, on constate qu'il s'agit d'une multitude de modèles qui visent à fournir des renseignements précieux sur les différentes manières avec lesquelles les individus apprennent (Gülbahar \& Alper, 2011: 271; Zywno et WaalenGlobal, 2002: 35). D'après Dodigovic (2005: 147), certains auteurs postulent que la majorité des classifications de styles d'apprentissage sont fondées sur la distinction entre les deux hémisphères cérébraux: droit et gauche. D'un point de vue anatomique, bien que les deux hémisphères soient 
très similaires, chacun assume des fonctions spécifiques. A titre d'illustration, l'hémisphère droit fonctionne plutôt sur la globalité, l'expérience, l'imagination, les émotions, la conscience de l'espace, etc., tandis que l'hémisphère gauche est censé être le siège du langage et de la logique qui accorde un type de traitement logique, mathématique, séquentiel, analytique, progressant du détail vers la complexité.

Or, d'autres modèles de classification existent. Pour Curry (1983: 8- 9) les modèles de styles d'apprentissage sont classifiés en fonction de trois groupes appartenant à différents niveaux. Le premier niveau, considéré comme le plus externe et le plus facilement observable, se rapporte aux préférences pour des conditions d'enseignement et d'apprentissage particulières telles que la luminosité de la pièce, le niveau de bruit ambiant, etc. En effet, ces caractéristiques, qui sont les plus sujettes à des influences contextuelles, s'avèrent les moins stables. Selon Chevrier et al. (2000a: 11), le modèle de Dunn \& Dunn fournit un bon exemple de ce niveau.

Quant au deuxième niveau, dit intermédiaire, il s'agit du style de traitement d'information qui convient aux caractéristiques des moyens privilégiés qu'adopte la personne afin d'assimiler l'information (Curry, 1983: 8- 9). En effet, ce niveau se caractérise, non seulement par la stabilité (en le comparant au premier niveau) causée par l'implication limitée de l'environnement, mais aussi par la fluctuation due aux stratégies d'apprentissage que choisissent les individus (Curry, 1983). Ce niveau est parfaitement représenté par les modèles de Kolb, Honey \& Mumford (Chevrier et al., 2000a) et de Fleming.

Le troisième niveau, vu comme le niveau interne le plus stable, renvoie au style de la personnalité cognitive. Celui-ci se réfère aux approches dont se sert l'individu afin d'adapter et d'assimiler les informations selon les traits de sa personnalité En fait, le modèle de Myers-Briggs constitue le meilleur exemple de ce niveau (Curry, 1983). 
Parmi les modèles de styles d'apprentissage les plus utilisés et les plus diffusés dans le monde de l'éducation, citons quelques-uns, à savoir: les modèles de Dunn \& Dunn, de Myers-Briggs et VAK de Fleming. Dans ce qui suit, nous aborderons brièvement chacun de ces modèles tout en mettant l'accent sur le modèle VAK de Fleming que la chercheuse a choisi afin de sous-tendre la présente étude vu sa simplicité et son usage fréquent dans différents domaines.

Premièrement, le modèle de Dunn \& Dunn. Dans ce modèle, Dunn \& Dunn suggèrent, au début, douze variables qu'ils jugent importantes pour l'apprentissage et qui peuvent être à l'origine des différences individuelles. Plus tard, ils ajoutèrent d'autres variables pour devenir enfin vingt et une réparties en cinq catégories, à savoir: les variables environnementales (telles que le son, la lumière et la température), les variables affectives (à l'exemple de la motivation, la persistance et la responsabilité), les variables sociologiques (concernant le fait d'apprendre mieux seul, avec un pair, en équipe, avec un adulte ou de manière variée), les variables physiologiques (sous forme de modalités perceptives efficaces (visuelle, auditive, tactile et kinesthésique) et de fluctuation du niveau d'énergie selon le moment de la journée, besoin de nourriture et de mobilité au cours de l'apprentissage) et les variables psychologiques (renvoyant au traitement global versus analytique, au degré de spécificité hémisphérique et au fonctionnement réfléchi versus impulsif) (Hlawaty, 2009: 25; Lovelace, 2005: 177-181; Chevrier et al., 2000c: 38).

Il est important de noter que Dunn \& Dunn affirment que personne n'est influencée par les vingt et un éléments tous à la fois. Ce sont environ six à quatorze éléments seulement qui peuvent affecter la majorité des individus (Dunn et al., 2001).

Deuxièmement, le modèle de Myers-Briggs (Aliweh, 2011: 83; Marquis, 1982: 44-47): en se basant sur la théorie des types de personnalité de Jung, Myers-Briggs classifie les individus en quatre catégories dont les combinaisons permettent de déterminer seize types de personnalité. Ces catégories sont: 
- Extraversion/ Introversion: la personne extravertie s'intéresse au monde extérieur, aux objets, aux gens et à l'action. Par contre, la personne introvertie accorde un soin particulier au monde intérieur des idées et de la contemplation.

- Sensation/Intuition: l'intérêt de l'individu sensoriel est dirigé vers l'immédiat, le réel, le tangible et les faits de l'expérience, par contre l'intuitif perçoit les possibilités, les relations et la signification des expériences tout en attribuant peu d'attention aux faits.

- Pensée/Emotion: une personne rationnelle prend des décisions d'une manière objective, impersonnelle tout en analysant et en ordonnant les faits, ce qui l'oppose à la personne émotive dont les décisions sont prises d'une façon subjective.

- Jugement/perception: la personne, qui préfère le jugement, aime vivre d'une manière planifiée et ordonnée afin de contrôler et d'uniformiser sa vie. En revanche, la personne perceptive aime vivre de façon spontanée et souple pour comprendre la vie et s'adapter aux différents événements.

Troisièmement, le modèle VAK (Visuel - Auditif - Kinesthésique) de Fleming qui constitue l'une des classifications des styles d'apprentissage les plus utilisées. Ce modèle est fondé principalement sur l'idée que nos styles d'apprentissage dépendent de notre traitement sensoriel des informations. Ce qui veut dire que les individus se servent de trois sens: la vue, l'ouïe et le toucher pour recevoir les informations, cependant, l'un de ces sens domine sur les deux autres (Alexander, 2011: 74; Reeder \& Joos, 2009: 67). Les styles d'apprentissage suggérés par Fleming sont au nombre de trois:

1) Le style visuel: l'apprenant visuel apprend très souvent en regardant. II préfère observer les gestes et les mimiques de son interlocuteur afin de mieux comprendre le message qui lui est transmis. Il apprend le mieux via des matériels visuels tels que les cartes, les graphiques, les vidéos, les documents, etc. De surcroît, lorsqu'il lit un texte, il opte pour une lecture silencieuse tout en surlignant, en couleur, les points importants qu'il renferme. En vue d'étudier, il privilégie les endroits tranquilles loin des 
perturbations verbales. Le style visuel se subdivise en deux souscatégories: linguistique et spatiale. Les individus, ayant un style visio-linguistique, saisissent mieux le contenu en lisant et en écrivant. Ils se souviennent de ce qu'ils ont lu même s'ils ne l'ont lu qu'une seule fois. Durant les cours magistraux, ils ont tendance à $\mathrm{y}$ assister et à prendre des notes détaillées. Par contre, les apprenants ayant le style visio-spatial éprouvent des difficultés avec le langage écrit. Ils préfèrent les graphiques, les démonstrations, etc. et ils imaginent facilement les visages et les endroits (Golon, 2008: 3-11).

2) Le style auditif: I'individu, ayant un style auditif d'apprentissage, apprend le mieux en écoutant. De même, il réagit bien pendant les cours magistraux, les discussions, les débats, les jeux de rôle, etc. II préfère les textes lus à haute voix aussi bien que les discours et les exposés en classe. Cette personne se parle souvent, bouge les lèvres pour absorber des renseignements, créé des petits refrains en vue de se mémoriser des connaissances apprises, relate des histoires pour éclaircir un point, etc. De plus, il a très souvent tendance à restituer le savoir tel qu'il était dit; il jouit donc d'une mémoire échoïque. En outre, la personne auditive est sensible aux sens implicites dégagés par le ton, la hauteur de la voix et le débit de l'orateur (Materna, 2007: 51; Tileston, 2004: 16-17; Muller, 2005: 351; Oxford, 2003: 4; Westen, 2000: 356).

3) Le style kinesthésique: une personne kinesthésique (tactile) apprend mieux dans des situations d'apprentissage pratiques telles que les expériences scientifiques et les activités de bricolage. Elle s'engage activement dans le processus de son apprentissage par la création des panneaux ou des cartes visant à consolider les connaissances apprises. Elle aime bouger et toucher les différents objets lorsqu'elle apprend de nouvelles choses. Par conséquent, elle devient facilement distraite à l'absence de toute stimulation externe ou si elle n'a pas le droit de changer de place pour une longue durée. Lors d'un cours magistral, l'apprenant kinesthésique aime prendre des notes, 
qu'il embellit d'illustrations ou de griffonnages. II préfère survoler le document écrit, au départ, afin d'avoir une idée générale avant de se concentrer sur les détails. De même, pour ce type d'apprenant, les pauses fréquentes lui semblent indispensables; elles l'aident à assimiler les notions apprises (Materna, 2007: 52; Tileston, 2004: 17- 18).

A partir de cette vue panoramique de quelques modèles de styles d'apprentissage, nous pouvons constater que chaque personne peut avoir un style spécifique ou une combinaison de styles, chose qui peut être mise en relief via les différents questionnaires (Martin et al., 2011: 361 - 363). En d'autres termes, les divers styles d'apprentissage sont étroitement liés puisque chaque personne peut avoir un mélange de style; toutefois, cela n'empêche pas la dominance d'un style sur les autres. Or, quel effet exercent les divers styles d'apprentissage sur la performance des futurs enseignants du FLE en ce qui concerne la compréhension orale? Question à laquelle la chercheuse tentera de répondre dans la partie suivante.

\section{Méthodologie}

Dans la présente étude, la chercheuse a pris appui tant sur la méthode descriptive que sur la méthode quasi - expérimentale. Le recours à la méthode descriptive était pour élaborer le cadre théorique autour des styles d'apprentissage. Quant à la méthode quasi-expérimentale, elle nous a permis d'identifier l'impact qu'exercent les divers styles d'apprentissage des futurs enseignants du FLE (la variable indépendante) sur la compréhension de l'oral (variable dépendante).

Cette partie de l'étude se subdivise en les sous - parties suivantes:

\section{a. Participants}

Les participants à l'étude ont été sélectionnés parmi les étudiants du diplôme général, section française. Ceux qui ont témoigné un enthousiasme pour participer à cette étude étaient au nombre de trente étudiants ( $80 \%$ de filles et $20 \%$ de garçons). L'âge moyen de ces participants était de 24.1 ans. Ils ont tous appris le français comme première langue étrangère depuis plus de quinze ans. De plus, ils sont tous licenciés de la faculté des Lettres, section 
française, et ils n'ont jamais eu l'expérience de travailler comme enseignants. Or, il est important de noter qu'on ne peut pas généraliser les résultats auxquels on aboutira sur l'ensemble des futurs enseignants du FLE à la faculté de Pédagogie d'Alexandrie. Le cas échéant, l'on est mené à déduire qu'il ne s'agit pas d'un échantillon représentatif des caractéristiques de toute la population vu le nombre restreint des participants.

\section{b. Instrumentation}

La collecte de données dans la présente étude est fondée sur l'usage de deux outils: un questionnaire autour des styles d'apprentissage basé sur le modèle VAK de Fleming et un test d'écoute. Voici une présentation plus ou moins détaillée de chacun de ces outils. Commençons par le questionnaire basé sur le modèle VAK qui vise à identifier le style d'apprentissage dominant chez l'échantillon de l'étude. La chercheuse a adopté le questionnaire élaboré par le centre Fora en collaboration avec le collège Boréal (2012). Celui-ci se compose de trente items. Il faut bien signaler qu'on a administré une version préliminaire du questionnaire sur neuf étudiants de la quatrième année, section française, à la faculté de Pédagogie d'Alexandrie afin de juger la clarté des différentes phrases et de s'assurer que ces phrases représentent les différents styles d'apprentissage. Vu que, dans la version originale, chaque item est succédé de trois phrases dont chacune renvoie à un style d'apprentissage: la première au style visuel, la deuxième au style auditif et la troisième au style kinesthésique, la chercheuse a dû changer l'ordre de ces phrases afin d'inciter les étudiants à réfléchir à toutes les phrases proposées sans les choisir automatiquement. D'après les résultats de cette étude pilote, la chercheuse a dû modifier certains items du questionnaire afin de les rendre plus clairs auprès des participants à l'étude (cf. Annexes).

Quant au test d'écoute, il avait pour but de tester l'habileté langagière concernant la compréhension orale chez les futurs enseignants du FLE. Ce test est adopté via un site ${ }^{1}$ qui prépare les apprenants aux tests internationaux tels que le DALF. Ce test, qui

${ }^{1}$ www.ciel.fr/apprendre-francais/preparation-examen/delf-b1-co.swf 335 
convient au niveau $\mathrm{B}^{2}{ }^{2}$, renferme dix questions de choix multiples. Ces dernières se rapportent à une séance d'écoute, de trois minutes environ, traitant les $C D$ en tant que support numérique (Perrot, s.d.). Dans ce test, un point est réservé pour chaque bonne réponse, tandis que le zéro est pour toute réponse erronée. Il faut bien souligner que la dernière question est sur quatre points parce qu'elle exige le choix de quatre bonnes réponses. Le test est donc sur treize points. De plus, la chercheuse a élaboré un modèle de réponse pour le test de l'étude (cf. Annexes).

\section{c. Déroulement}

Afin d'atteindre les objectifs de l'étude, la chercheuse a utilisé un protocole de recherche quantitative accompagné d'une analyse de variance à un facteur (ANOVA). Dans un premier temps, les futurs enseignants ont été interrogés sur leurs styles d'apprentissage par l'intermédiaire d'un questionnaire fondé sur le modèle VAK de Fleming. Dans un deuxième temps, on a appliqué le test d'écoute sur l'échantillon de l'étude dans le but de tester leur niveau de performance à l'habileté langagière en question. Il faut bien noter que l'administration du questionnaire et du test, qui a duré environ 20 minutes pour chacun, a eu lieu dans le cadre du deuxième semestre de l'année académique 2014/ 2015. A la lueur des résultats issus du questionnaire, la chercheuse a classifié les futurs enseignants en trois groupes (visuels, auditifs et kinesthésiques) en fonction de leurs styles d'apprentissage. De surcroît, après avoir calculé les notes des participants sur le test d'écoute, la chercheuse s'est servie de I'ANOVA pour investiguer l'effet qu'exerce la variable indépendante, à savoir les styles d'apprentissage et ses différents types sur la variable dépendante, c'est-à-dire la performance des futurs enseignants du FLE sur le test d'écoute.

\section{d. Méthode d'analyse des données}

La chercheuse a eu recours à I'ANOVA qui semble la technique statistique la plus propice (Haccoun \& Cousineau, 2007: 329 - 330) à cette étude puisqu'elle permet de déterminer l'impact qu'exercent

${ }^{2}$ C'est -à - dire un niveau intermédiaire. 
les différents styles d'apprentissage sur la performance des futurs enseignants du FLE sur le test d'écoute. Afin de nous assurer que les données sont conformes à ce type d'analyse, la chercheuse a vérifié, en premier lieu, la normalité et l'homogénéité des variances; conditions indispensables pour lancer l'ANOVA. En effet, la première condition a été vérifiée à l'aide des tests de Kolmogorov-Smirnov et de Shapiro-Wilk. Les résultats de ceux -ci mettent en évidence que les notes des futurs enseignants du FLE sur le test de performance suivent la distribution normale. Quant à la deuxième condition concernant l'homogénéité des variances, elle a été examinée via le test de Levene qui est le plus satisfaisant pour s'en assurer (Gauvrit, 2006: 67). Il est vraisemblable que l'application de ce test montre que le résultat n'est pas statistiquement significatif (Sa valeur $>0.05$ ), ce qui prouve l'homogénéité des variances. II s'agit en, deuxième lieu, de calculer la moyenne arithmétique, l'écart - type, le minimum et le maximum des notes des futurs enseignants visuels, auditifs et kinesthésiques sur le test de performance portant sur l'écoute. Or, il faut souligner que la chercheuse a effectué cette analyse via le programme SPSS version 19. De même, on a interprété tous les résultats de l'étude à la lueur du seuil de 0.05 .

\section{e. Considérations éthiques}

Uniquement trente futurs enseignants du FLE inscrits au diplôme général, section française, ont consenti à participer à la présente étude. La chercheuse les a informés qu'ils ont le droit de s'y retirer à n'importe quel moment, sans encourir aucun préjudice. En vue de respecter leur confidentialité, on leur a demandé de remplacer leurs noms, par un symbole ou un pseudonyme. Avant l'application des outils de l'étude, la chercheuse a clarifié, auprès des participants, les objectifs de ce recueil de données. Une semaine après la passation du test et du questionnaire, la chercheuse leur a présenté les résultats de l'étude.

\section{Résultats}

Parmi les trente futurs enseignants du FLE, $50 \%$ ont un style auditif, $40 \%$ ont un style visuel et $10 \%$ ont un style kinesthésique. Ceux ayant un style auditif ont eu les meilleures notes sur le test 
d'écoute. Ceci semble clairement par l'intermédiaire de la moyenne arithmétique (5.13). Néanmoins, les futurs enseignants ayant le style visuel ont obtenu les notes les plus basses au test en question. La moyenne arithmétique était 2.75 . Le tableau suivant met en exergue ces résultats.

Tableau 2 Statistique descriptive des notes des futurs enseignants du FLE ayant différents styles d'apprentissage

\begin{tabular}{|c|c|c|c|c|c|}
\hline $\begin{array}{c}\text { Futurs } \\
\text { enseignants }\end{array}$ & $\begin{array}{c}\text { Nombre } \\
\text { d'étudiants }\end{array}$ & $\begin{array}{c}\text { Moyenne } \\
\text { arithmétique }\end{array}$ & $\begin{array}{c}\text { Écart- } \\
\text { type }\end{array}$ & $\begin{array}{c}\text { Minimum } \\
\text { des notes }\end{array}$ & $\begin{array}{c}\text { Maximum } \\
\text { des notes }\end{array}$ \\
\hline Visuels & 12 & 2.75 & 1.60 & 0 & 6 \\
\hline Auditifs & 15 & 5.13 & 2.50 & 2 & 11 \\
\hline Kinesthésiques & 3 & 3.33 & 1.15 & 2 & 4 \\
\hline
\end{tabular}

En vue d'examiner l'effet qu'exerce le style d'apprentissage sur la performance des étudiants au test d'écoute, la chercheuse a mis en œuvre I'ANOVA. En effet, l'application de ce modèle statistique montre clairement que la valeur du $F$ calculée dépasse celle du tableau statistique au niveau de 0.05 , ce qui prouve qu'il y a des différences statistiques significatives dans la performance des futurs enseignants en fonction de leurs styles d'apprentissage. Le tableau cidessous illustre ce résultat.

Tableau 3 Résultats de l'ANOVA

\begin{tabular}{|c|c|c|c|c|}
\hline $\begin{array}{c}\text { Sources de } \\
\text { variation }\end{array}$ & $\begin{array}{c}\text { Somme des } \\
\text { écarts }\end{array}$ & $\begin{array}{c}\text { Degrés de } \\
\text { liberté }\end{array}$ & Carré moyen & F \\
\hline Inter & 39.35 & 2 & 19.67 & 4.48 \\
\hline Intra & 118.65 & 27 & 4.39 & \\
\hline Total & 158.00 & 29 & & \\
\hline
\end{tabular}

Afin d'identifier les groupes qui sont particulièrement différents les uns des autres, il s'avère indispensable d'effectuer des comparaisons a posteriori (post-hoc) et précisément le test de Tukey. Ce dernier prouve qu'il s'agit des différences significatives, uniquement, entre la performance des futurs enseignants auditifs en compréhension de l'oral et celle des visuels en faveur des premiers.

\section{f. Discussion des résultats}

Cette étude exploratoire visait à connaître l'effet des styles d'apprentissage sur la performance des futurs enseignants du FLE en 
compréhension orale. A la lueur des résultats auxquels nous avons abouti, il paraît clairement que la performance des futurs enseignants du FLE en compréhension de l'oral était influencée par leurs styles d'apprentissage. Ceux ayant un style auditif ont obtenu les meilleures notes au test de la compréhension orale, ce qui n'était pas le cas avec ceux des styles visuel ou kinesthésique. En effet, ce résultat convient parfaitement avec ceux de Liu (2008) et de Bidabadi \& Yamat (2012) qui soulignent que le style communicatif d'apprentissage, selon le modèle de Willing, (correspondant au style auditif dans cette étude) fournit un indicateur positif de la performance des apprenants en compréhension de l'oral.

\section{Conclusion et recommandations}

En guise de conclusion, il est incontestable que cette étude a été accomplie en vue d'investiguer l'impact qu'exercent les styles d'apprentissage des futurs enseignants du FLE sur leurs performances en compréhension de l'oral. Afin d'identifier cet impact, la chercheuse s'est servie de I'ANOVA suivie du test de Tukey. Les résultats de ces techniques statistiques mettent en relief qu'il s'agit des différences statistiques significatives dans la performance des futurs enseignants en faveur de ceux ayant un style auditif. En d'autres termes, le style auditif exerce un effet crucial sur la performance des futurs enseignants du FLE en ce qui concerne l'écoute. Ce qui explique la raison pour laquelle les notes des futurs enseignants auditifs sont plus élevées par rapport à leurs collègues visuels ou kinesthésiques.

En fonction des résultats auxquels nous sommes parvenus, il s'avère indispensable de tenir compte des styles d'apprentissage des étudiants lors de la passation des tests standardisés de performance. C'est pourquoi une nouvelle vision de ces tests, fondée sur la prise en considération des styles d'apprentissage, semble primordiale.

Par ailleurs, vu le nombre restreint de l'échantillon, la chercheuse recommande l'accomplissement d'autres études avec un grand nombre de participants afin de pouvoir, d'une part confirmer les constatations auxquelles on est parvenue, et d'autre part les généraliser pour en profiter ultérieurement. 
De surcroît, une étude qualitative approfondie des stratégies dont se servent les étudiants ayant un style auditif nous permettra de mieux comprendre comment procèdent ceux-ci lors des tests de performance portant sur l'écoute. 


\section{Bibliographie}

1. Ahmad, A. (2011). Language learning style preferences of low english proficiency (LEP) students in a tertiary institution. Malaysian journal of ELT research, 7(2), 33 - 62.

2. Alexander, L. (2011). How to incorporate wellness coaching into your therapeutic practice: a handbook for therapists and counselors. Londres: Singing dragon.

3. Aliweh, A. (2011). Exploring Egyptian EFL students' learning styles and satisfaction with web-based materials. CALICO Journal, 2(1), 81-99.

4. Arthur J. (1993). Learning styles and the classroom. Récupéré du site: https://eric.ed.gov/?id=ED368479.

5. Atlan, J. (2000). L'utilisation des stratégies d'apprentissage d'une langue dans un environnement des TICE. Alsic, 3(1), juin, 109 123. Récupéré du site de la revue: http://alsic.revues.org/1759?

6. Barmeyer, C. (2007). Management interculturel et styles d'apprentissage: étudiants et dirigeants en France, en Allemagne et au Québec. Les presses de l'université Laval.

7. Bidabadi, F. \&Yamat, H. (2012). The Relationship between English Listening Proficiency levels and learning styles. GEMA Online ${ }^{T M}$ Journal of language studies, 12(4), novembre, 1041 - 1055. Récupéré du site: http://ejournal.ukm.my/gema/article/view/1568/1355.

8. Bouchafa, H. (2009). Styles cognitifs dans le traitement de I'expérience. $A B C$ de la VAE. Toulouse: Erès, 217-218. Récupéré du site: http://www.cairn.info/l-abc-de-la-vae--9782749211091.

9. Brown, H. (2000). Principles of language teaching and learning $\left(4^{\mathrm{e}}\right.$ édition). White Plains, New York: Longman.

10. Buck, G. (2001). Assessing listening. Cambridge: Cambridge University Press.

11. Chartier, D. (2003). Les styles d'apprentissage: entre flou conceptuel et intérêt pratique. Savoirs, 2(2), 7-28. 
12. Centre Fora en collaboration avec le collège Boréal. (2012). Styles d'apprentissage. Récupéré du site:

http://centrefora.on.ca/sites/default/files/documents/Ressource s/Styles\%20d\%27apprentissage.pd.

13. Chevrier, J., Fortin G., Leblanc R. \& Théberge, M. (2000a) Problématique de la nature du style d'apprentissage. Education et francophonie. printemps, 28, 3-19.

14. Chevrier, J., Fortin G., Leblanc R. \& Théberge, M. (2000b). La construction du style d'apprentissage. Education et francophonie. printemps, 28,47-72.

15. Chevrier, J., Fortin, G., Théberge M. \& Leblanc, R. (2000c). Le style d'apprentissage: une perspective historique. Education et francophonie. printemps, 28, $20-46$.

16. Coffield, F. (2004). Learning styles and pedagogy in post-16 learning: a systematic and critical review. Londres: Learning and skills research centre.

17. Cornett, C. (1983). What you should know about teaching and learning styles. Bloomington, Indiana: Phi delta kappa educational foundation.

18. Cox, T. (2008). Learning styles and students' attitudes toward the use of technology in higher and adult education classes. Institute for learning styles journal, 1, automne, 1-13.

19. Curry, L. (1983). An organization of learning style theory and constructs. Paper presented at the annual meeting of the american educational research association. 67th, Montréal, Québec, avril, 11-15.

20. Denig, S. (2004). Multiple intelligences and learning styles: Two complementary dimensions. Teachers college record, 106(1), 96111.

21. Dennis, M. (2002). Applying what we know: student learning style. Récupéré du site: http://www.ctserc. org/library/bibfiles/learning-styles.pd.

22. Dodigovic, M. (2005). Artificial intelligence in second language learning: raising error awareness. Toronto, Canada: Multilingual Matters Ltd. 
23. Dörnyei, Z. \& Skehan, P. (2003). Individual differences in second language learning. In C. J. Daughty \& M. H. Long (Eds.), The handbook of second language acquisition (pp.589-630). Oxford, United Kingdom: Blackwell Publishing LTD.

24. Dunn R, Denig, S. \& Lovelace, M. (2001). Two sides of the same coin or different strokes for different folks? Teacher librarian, 28(3), 9-15.

25. Fleming, N. \& Baume, D. (2006) Learning Styles Again: VARKing up the right tree! Educational Developments, SEDA Ltd, 7(4), novembre, 4-7.

26. Flessas, J. (1997). L'impact du style cognitif sur les apprentissages. ACELF: Les difficultés d'apprentissage, 25(2), automne-hiver, Récupéré du site de la revue: http: // www.acelf.ca/c/revue/revuehtml/25-2/r252-03.html.

27. Franzoni, A. \& Assar, S. (2009). Student learning styles adaptation method based on teaching strategies and electronic media. Educational technology and society, 12(4), 15-29.

28. Frayssinhes, J. (2011). Les pratiques d'apprentissage des adultes en FOAD: effet des styles et de l'auto-apprentissage (thèse de doctorat non publiée). Université de Toulouse II - le Mirail, France.

29. Gagnon, R., De Pietro, J.-F. \& Fisher, C. (2017). L'oral aujourd'hui: perspectives didactiques (introduction). In: J.-F. de Pietro, C. Fisher \& R. Gagnon (Eds.), L'oral aujourd'hui: perspectives didactiques (pp. 11-40). Namur: Presses universitaires de Namur. Récupéré du site: https://www.irdp.ch/data/secure/2493/document/depietro_oral_auj ourdhui.pdf

30. Gardner, H. (1999). Intelligence reframed. Multiple intelligences for the 21st century. New York: Basic Books.

31. Gaussel, M. (2013). Apprentissage: à chacun son style ? Entretien avec Marie Gaussel. Cahiers pédagogiques, 506, juin. Récupéré du site de la revue: http://www.cahiers-pedagogiques.com/Apprentissage-a-chacunson-style. 
32. Gauvrit, N. (2006). Stats pour psycho. Bruxelles, Belgique: de Boeck.

33. Gilakjani, A. (2012). Visual, auditory, kinesthetic learning styles and their impacts on english language teaching. Journal of studies in education, 2(1), 104 -113.

34. Gilakjani, A. \& Ahmadi, S. (2011). The effect of visual, auditory, and kinesthetic learning styles on language teaching. International conference on social science and humanity IPEDR, 5, $469-472$.

35. Golon, A. (2008). Visual-spatial learners: differentiation strategies for creating a successful classroom (2e édition). Waco, TX: Prufrock press.

36. Guild, P. (1997). Where do the learning Theories overlap? Education leadership, 55(1), 30-31.

37. Gülbahar, Y. \& Alper, A. (2011). Learning preferences and learning styles of online adult learners. In: A. Méndez-Vilas (Ed.), Education in a technological world: communicating current and emerging research and technological efforts (pp.270 - 278). Bendajoz: Formatex research center.

38. Haccoun, R. \& Cousineau, D. (2007). Statistiques: concepts et applications. Montréal, Québec: Presses de l'université de Montréal.

39. Hanachi- Ferhoune, N. (2007). Stratégies d'apprentissage développées par les collégiens et les lycéens en classe de langue (thèse de maîtrise non publiée). Université Mentouri Constantine.

40. Hlawaty, H. (2009). Lernen and learning styles: a comparative analysis of the learning styles of German adolescents by age, gender, and academic achievement level. European education, 40(4), hiver, 23-45, Récupéré du site: http://web.a.ebscohost.com/ehost/detail?sid=45160e23-bec646b0-9e0ba970512e354b\%40sessionmgr4004etvid=6ethid .

41. Karthigeyan, K. \& Nirmala, K. (2013). Learning style preference of english language learners. Educationia confab, 2(1), janvier, $134-140$. 
42. Kassaia, Z. (2007). Learning styles and lexical presentations modes. Iran Estudios de lingüística inglesa aplicada (Elia), $53-78$

43. Lavine, G. \& Fiala, Y. (2006). Influence du style d'apprentissage (intelligences multiples) sur l'apprentissage de l'expression orale en anglais langue seconde au collégial. Etude menée dans le cadre du programme de recherche et développement du réseau privé de l'enseignement collégial. Récupéré du site : http://www.cadre.qc.ca/.../Rapport\%20rech_Influence\%20du\%20 style \%.

44. Liu, H.-J. (2008). A study of the interrelationship between listening strategy use, listening proficiency levels, and learning style. ARECLS, 5, 84-104.

45. Lovelace, M. (2005). Meta-Analysis of experimental research based on the Dunn and Dunn model. The journal of educational research, 98 (3) janvier - février, 176-183

46. Marquis, J.-P. (1982). L'analyse du style d'apprentissage: Modèle et instrument (thèse de maîtrise non publiée). Centre d'études universitaires d'Abitibi- Temiscamingue, université du Québec.

47. Martin, H., Lewis, T. \& Edwards, K. (2011). Leadership, learning styles and academic performance of undergraduate engineering students in Trinidad. World engineering education (WEE), septembre 27-30, $359-364$.

48. Materna, L. (2007). Jump-start the adult learner: how to engage and motivate adults using brain - compatible strategies. California: Corwin press.

49. Matthews, D. (1996). An investigation of learning styles and perceived academic achievement for high school students. The Clearing House, 69(4), mars - avril, 249-254.

50. Muller, F. (2005). Manuel de survie à l'usage de l'enseignant (même le débutant). Paris: L'Etudiant.

51. O'Malley, J. \& Chamot, A. (1995). Learning strategies in second language acquisition ( $4^{\mathrm{e}}$ édition). Cambridge: Cambridge University Press.

52. Oxford, R. (1990). Language learning strategies: What every teacher should know. Boston: Heinle, Cengage learning. 
53. Oxford, R. (2003). Language learning styles and strategies: an overview. Learning styles and strategies, Oxford, GALA, 125. Récupéré du site: http://hyxy.nankai.edu.cn/.../learning\%20strategies\%20by\%200xf ord.pdf.

54. Oxford, R. \& Lavine, R. (1992). Teacher-Student style wars in the Language classroom: research insights and suggestions. ADFL bulletin, 23(2), hiver, 38-45, Récupéré du site: http://www.adfl.org/bulletin/V23N2/232038.HTM.

55. Özgen, K., Tataroğlu, B \& Alkan, H. (2011). An examination of multiple intelligence domains and learning styles of preservice mathematics teachers: their reflections on mathematics education. Educational research and reviews, 6(2), février,168181.

56. Penger, S. \& Tekavčič, M. (2009). Testing Dunn \& Dunn's and Honey \& Mumford's learning style theories: the case of the Slovenian higher education system. Management, 14(2), 1-20.

57. Perrot, A. (s.d.).Online listening comprehension exercises level B1: CD life. Le Français dans le monde, Récupéré du site: http://www.ciel.fr/apprendre-francais/preparationexamen/comprehension-oral b1.html.

58. Prashnig, B. (2005). Learning styles vs. multiple intelligences (MI) two concepts for enhancing learning and teaching, 9, automne, 89. Récupéré du site: http:// www.teachingexpertise.com.

59. Raven, M., Cano J., Carton B \& Shelhamer V. (1993). A comparison of learning styles, teaching styles, and personality styles of preservice Montana and Ohio agriculture teachers. Journal of agricultural education, printemps, $40-50$.

60. Reeder, F. \& Joos, A. (2009). Fire service instructors: principles and practice ( 2 édition). Londres: Jones et Bartlett.

61. Rost, M. (2011). Teaching and researching listening ( $2^{\mathrm{e}}$ édition.). Harlow, UK: Pearson.

62. Rey, A. Le Robert Micro poche. (1997). Montréal, Québec: Dico Robert Inc. 
63. Rieben, L. (2000). A quelles conditions la notion de style d'apprentissage peut-elle devenir heuristique pour le champ de l'éducation? ACELF. printemps, 28 (1), 136-147.

64. Shi, C. (2011). A study of the relationship between cognitive styles and learning strategies. Higher education studies, 1(1), juin, 2026.

65. Silver, H., Strong, R. \& Perini, M. (1997). Integrating learning styles and multiple intelligences. Education Leadership, 55(1), 2227.

66. Smith, P. \& Dalton, J. (2005). Getting to grips with learning styles. Adelaide: NCVER.

67. Therer, J. (1998). Styles d'enseignement, styles d'apprentissage et pédagogie différenciée en sciences. Informations pédagogiques, 40, mars, 1-23.

68. Tileston, D. (2004). What every teacher should know about learning, memory, and the brain. California, USA: Corwin press.

69. Torresan, P. (2010). Théorie des intelligences multiples et apprentissage des langues.

70. Perugia: Guerra Edizioni. (Traduction de Dominique Paravel). Récupéré du site: http://arcaold.unive.it/bitstream/10278/2318/1/Nr.\%206\%20versio ne\%20francese.pdf

71. Tremblay, M. \& Paradis, J. (2001). L'importance du style d'apprentissage en supervision. Bulletin de la formation clinique. Université d'Ottawa. septembre, 3-6.

72. Wang, J. (2015). L'impact des stratégies et styles d'apprentissage sur le sentiment de réussite ou d'échec dans l'apprentissage de langues étrangères. Cahiers de l'Apliut, 34(2). Récupérédu site: https://journals.openedition.org/apliut/5223

73. Westen, D. (2000). Psychologie : pensée, cerveau et culture. Paris: De Boeck université. 
74. Zainol Abidin, M., Rezaee, A., Abdullah, H. \& Singh, K. (2011). Learning styles and overall academic achievement in a specific educational system. International journal of humanities and social science, 1, (10), août, 143- 152. Récupéré du site: http://www.ijhssnet.com.

75. Zywno, M. \& WaalenGlobal, J. (2002). The effect of individual learning styles on student outcomes in technology-enabled education. Global journal of engineering education, 6(1), 35 - 44.

76. www.ciel.fr/apprendre-francais/preparation-examen/delf-b1co.swf. 